\title{
Scratch Detection by Eddy-Current Testing with a High Lift-Off Height
}

\author{
H. Tian, S. Yamada, M. Iwahara, and H. Watanabe* \\ Kanazawa University, 2-4-20 Kodatsuno, Kanazawa 920-8667, Japan \\ * Daido Steel Co.Ltd., 2-30 Daido-cho, Nagoya 457-8546, Japan
}

This paper describes an eddy-current testing (ECT) probe with a new structure, consisting of a perpendicular exciting coil and a spin-valve-type giant magneto-resistance sensor for scratch detection with a high lift-off height. We derived an analytical solution of eddy-current distribution to compare with a perpendicular exciting coil and a traditional parallel exciting coil. Experimental inspections with lift-off heights of 5 and $7 \mathrm{~mm}$ were carried out to detect scratches of $0.1-0.5$ $\mathrm{mm}$ depth in a stainless steel plate. The 2-D experimental image results show that the proposed ECT probe has good detection capabilities.

Key words: eddy currents, giant magneto-resistance, perpendicular coil, scratch, lift-off height

\section{Introduction}

Scratch inspection with a high lift-off height in eddycurrent testing (ECT) has wide and important applications, for instance, preventing probe from a high temperature damage in the heat treatment status of metals or inspecting embedded metal particles without surgical operation in biomedical field, etc. However, most of reliable ECT detections are carried out in intimate contact with the material being tested or with a low lift-off height because it is a knotty detection in the high lift-off situation.

In the case of a high lift-off height, induced eddy currents attenuate in terms of exponential function. Consequently, the secondary magnetic field produced by attenuated eddy currents also is weaken so that the detected magnetic signals originated from scratches become weak more. In addition, as the probe is located in a high lift-off position, the distance between probe and surface of a detected material is tended to make the secondary magnetic signals suffer from acute attenuation again. The previous paper said that it is difficult to keep a high lift-off height by the ECT probe consisting of a traditional parallel style exciting coil and a spin-valve-type giant magneto-resistance (SV-GMR) ${ }^{1}$.

In this paper, we offer an approximate analytical solution with respect to a rectangular exciting coil perpendicular to a plane. According to the analytic solution, we calculated the eddy-current distribution and compared it with a traditional parallel style of a circular exciting coil. The evaluation of an eddy-current distribution and SV-GMR sensor's property enable us to propose a new ECT probe for scratch detection with a high lift-off height.

Furthermore, experiments were carried out to detect a series of scratches with $0.1-0.5 \mathrm{~mm}$ depth in a stainless steel (SS) plate. The 2-D image results demonstrate the ability of probe in detecting shallow scratches with lift-off heights of 5 and $7 \mathrm{~mm}$.

\section{Analytical solutions of perpendicular and parallel exciting coil}

\subsection{Rectangular coil perpendicular to a plane}

For an arbitrary orientation point current source (PCS) paralleling to a plane with a conductivity $\sigma$ and a permeability $\mu=\mu_{r} \mu_{0}$, and a carrying current $I e^{j \omega t}$ with a length $d l$, it can be decomposed parallel component $d l_{/ /}$and perpendicular component $d l_{\perp}$ as shown in Fig. 1, which solutions response to vector potential $d A_{/ / 2}$ and $d A_{\perp}$ as follows with $\mu_{r}=1$ (stainless steel) $^{2)}$,

$$
\begin{aligned}
d A_{/ /}^{\text {metal }}= & \frac{\mu_{0} I d l_{\| /}}{2 \pi} \cdot \int_{0}^{+\infty} \alpha J_{0}(\alpha r) e^{-\alpha h} \\
& \times \frac{\left(\alpha+\alpha_{m}\right) e^{\alpha_{m} z}+\left(\alpha-\alpha_{m}\right) e^{-\alpha(2 d+z)}}{\left(\alpha+\alpha_{m}\right)^{2}-\left(\alpha-\alpha_{m}\right)^{2} e^{-2 d \alpha_{m}}} d \alpha \cdot e_{x} \\
d A_{\perp}^{\text {metal }}= & \frac{\mu_{0} I d l_{\perp}}{2 \pi} \cdot \int_{0}^{+\infty} \alpha J_{1}(\alpha r) e^{-\alpha h} \\
& \times \frac{\left(\alpha+\alpha_{m}\right) e^{\alpha_{m} z}+\left(\alpha_{m}-\alpha\right) e^{-\alpha_{m}(2 d+z)}}{\left(\alpha+\alpha_{m}\right)^{2}-\left(\alpha-\alpha_{m}\right)^{2} e^{-2 d \alpha_{m}}} d \alpha \cdot u_{r}
\end{aligned}
$$

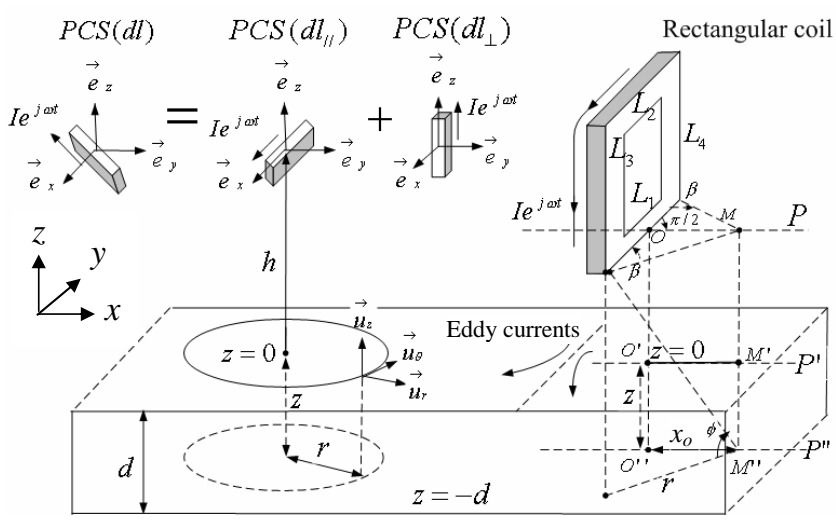

Fig. 1 Rectangular coil perpendicular to a plane. 


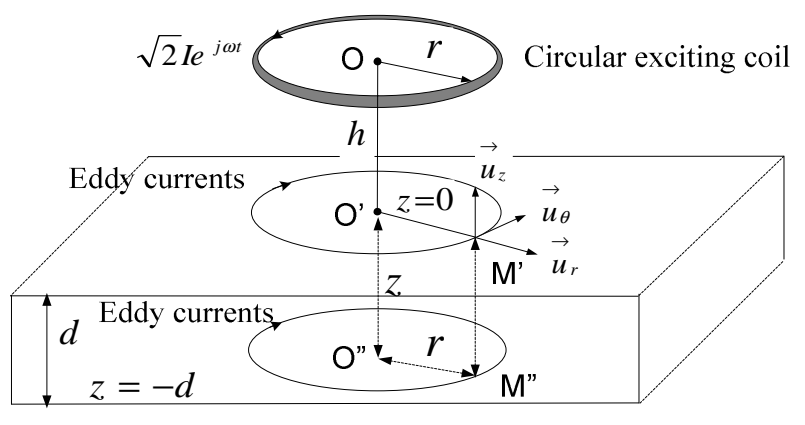

Fig. 2 Circular exciting coil parallel to a plane.

where

$$
\alpha_{m}=\sqrt{\alpha^{2}+j \omega \mu_{0} \mu_{r} \sigma}
$$

and, $J_{0}$ and $J_{1}$ are the zero- and first-order Bessel function of the first kind.

In the case of a rectangular coil perpendicular to a plane in Fig. 1 (right), it consists of four pieces of currentcarrying wire of infinite length. We integrate Eqs. (1) and (2) at any point $M^{\prime \prime}$ on the axis $P^{\prime \prime}$ (O is the center point of line $L_{1}, P^{\prime}$ and $P^{\prime \prime}$ are projections of $P$ axis.), and in terms of Eq. (4),

$$
J(r, z)=\sigma E=-\sigma \partial A / \partial t=-j \omega \sigma A
$$

then the solutions of eddy currents induced by parallel lines $L_{1}$ and perpendicular lines $L_{3}$ are derived as follows respectively,

$$
\begin{gathered}
J_{/ /}(r, z)=-j \omega \sigma \mu_{0} I / \pi \cdot \int_{n_{0}}^{\sqrt{\left.\left(L_{1} / 2\right)^{2}+r_{0}^{2}\right)}} \frac{r}{\sqrt{r^{2}-r_{0}^{2}}} \cdot\left\{\int_{0}^{+\infty} \alpha J_{0}(\alpha r) e^{-\alpha h}\right. \\
\left.\times \frac{\left(\alpha+\alpha_{m}\right) e^{\alpha_{m} z}+\left(\alpha_{m}-\alpha\right) e^{-\alpha_{m}(2 d+z)}}{\left(\alpha+\alpha_{m}\right)^{2}-\left(\alpha-\alpha_{m}\right)^{2} e^{-2 d \alpha_{m}}} \cdot d \alpha\right\} \cdot d r \cdot e_{x}
\end{gathered}
$$

$$
\begin{aligned}
J_{\perp}(r, z) & =-j \omega \sigma \mu_{0} I / 2 \pi \cdot \cos \beta \cdot \int_{a c \tan (h+z) / r)}^{a c \tan \left(\left(h+z+l_{3}\right) / r\right)} r \sec ^{2} \phi\left\{\int_{0}^{+\infty} \alpha J_{1}(\alpha r) e^{-\alpha h}\right. \\
& \left.\times \frac{\left(\alpha+\alpha_{m}\right) e^{\alpha_{m} z}+\left(\alpha_{m}-\alpha\right) e^{-\alpha_{m}(2 d+z)}}{\left(\alpha+\alpha_{m}\right)^{2}-\left(\alpha-\alpha_{m}\right)^{2} e^{-2 d \alpha_{m}}} d \alpha\right\} \cdot d \phi \cdot e_{x}
\end{aligned}
$$

By superimposing the solutions of four lines $L_{1}, L_{2}, L_{3}$, and $L_{4}$, we can get the solution of eddy currents at point $M "$.

\subsection{Circular exciting coil parallel to a plane}

For a circular coil of radius $R$, carrying current $\sqrt{2} I \sin \omega t$, coplanar to a conductor plane of conductivity $\sigma$, permeability $\mu=\mu_{r} \mu_{0}$, and thickness $d$, this model has been proposed by Dodd and Deed ${ }^{3)}$. Here we give the analytical solution of eddy-current density in cylindrical coordinates $(r, \theta, z)$ directly, with $\mu_{r}=1$ (stainless steel),

$$
\begin{aligned}
J=- & j \omega \sigma \mu_{0} I R \cdot \int_{0}^{\infty} \alpha e^{-\alpha h} J_{1}(\alpha R) J_{1}(\alpha r) \\
& \frac{\left(\alpha+\alpha_{m}\right) e^{\alpha_{m}(2 d+z)}+\left(\alpha_{m}-\alpha\right) e^{-\alpha_{m} z}}{\left(\alpha+\alpha_{m}\right)^{2} e^{2 \alpha_{m} d}-\left(\alpha_{m}-\alpha\right)^{2}} d \alpha \cdot u_{\theta}
\end{aligned}
$$

\section{Comparison between perpendicular and parallel exciting coils}

The magnetic flux density induced by eddy currents on the surface layer plays an important role in the detection to shallow scratches. Consequently, it is significant to evaluate the eddy-current distribution on the surface layer of SS plate for each style of an exciting coil.

In order to compare the two style coils, we assume perpendicular square coil and parallel circular coil have the same length perimeter $\left(30\left(L_{1}\right) \times 4 \mathrm{~mm}\right)$. They are placed at lift-off height $h=5 \mathrm{~mm}$ above $d=12 \mathrm{~mm}$ thick SS304 plate $\left(\sigma=1.1 \times 10^{6} \mathrm{~S} / \mathrm{m}, \mu_{r}=1\right)$, supplied by current $I=100 \mathrm{~mA}$ with 100 turns and $f=50 \mathrm{kHz}$.

The eddy-current density distribution is variable related to depth $\mathrm{z}$ of SS plate and distance $r$ from the center point of coordinates system. The distributions of real component and imaginary component for each style of exciting coil are shown in Figs. 3 and 4.

In the case of a high lift-off height, a sensor is located at higher position than surface detection. The magnetic flux density at higher position is more susceptible to the spatial distribution of secondary magnetic fields generated by eddy currents flowing in the different position in SS plate. The total secondary magnetic field is a superposition of these magnetic fields. Therefore, the eddy-current distribution in surface layer is indispensable and has to be considered in scratch detection.

For a perpendicular exciting coil, both real component and imaginary component are the plus distribution in the surface layer of SS plate as shown in Fig. 3, which can generate the same direction secondary magnetic fields. The same direction magnetic fields will be superimposed to enhance the signal strength at spatial position oriented sensor.

However, for a parallel exciting coil, it produces serious minus distribution along the radial direction $r$ shown in Fig. 4. Both real and imaginary part present minus as well as plus eddy-current distribution in the surface layer of SS plate. This positive-negative eddy-current distribution will induce positive-negative magnetic fields. The positive-negative magnetic fields are conflicted each other at spatial point oriented sensor so that the total secondary magnetic field detected by sensor is weaken. 


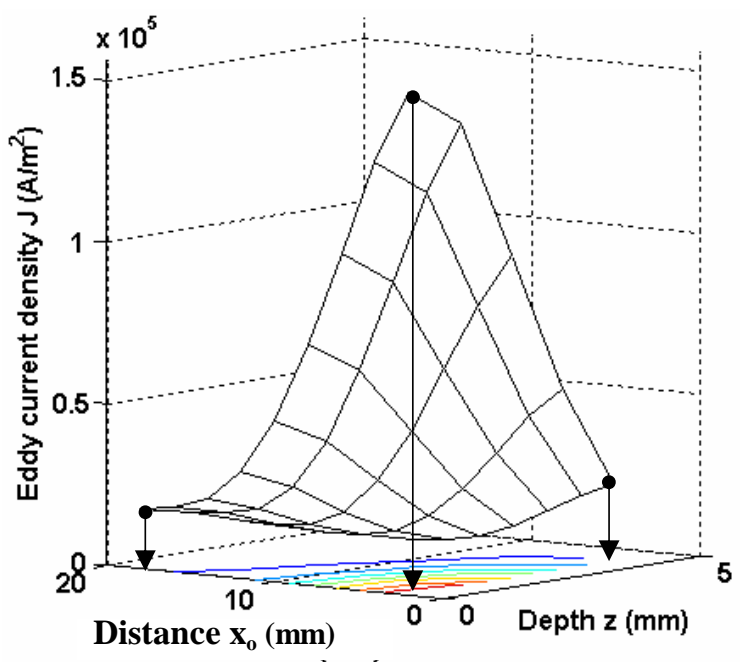

(a) Real component

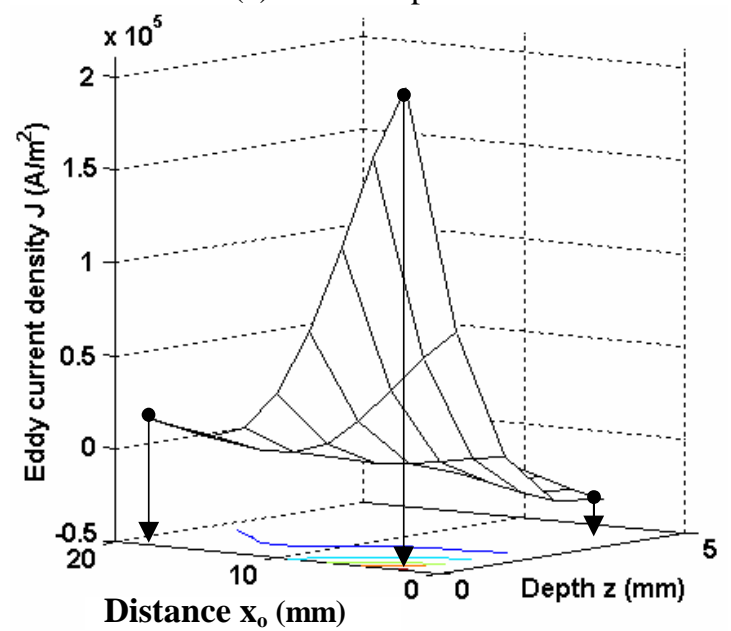

(b) Imaginary component

Fig. 3 Eddy-current distribution for a rectangular coil perpendicular to a plane.

This situation is not available especially when the detected signal is very weak such as in the case of high lift-off inspection.

Comparing with a traditional parallel style of exciting coil, a perpendicular style of exciting coil can provide eddy-current distribution with the same polarity in the surface layer, which induces an enhanced secondary magnetic field. Additionally, a perpendicular style of exciting coil also decreases the amount of air-couple field so that the lift-off noise is eliminated to a lower lever than parallel style of exciting coil. Thus, the perpendicular style exciting coil is designed in scratch inspection with high lift-off height.

\section{ECT probe design}

The fabrication of ECT probe including SV-GMR sensor properties and the tested specimen are shown in Fig.5.

SV-GMR sensor is setup on the axis $\mathrm{P}$, on which $z$ -

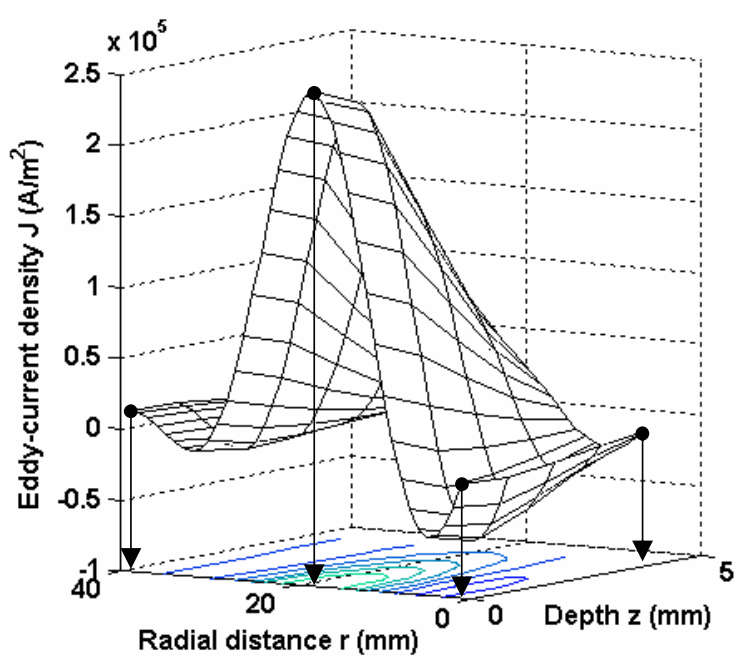

(a) Real component

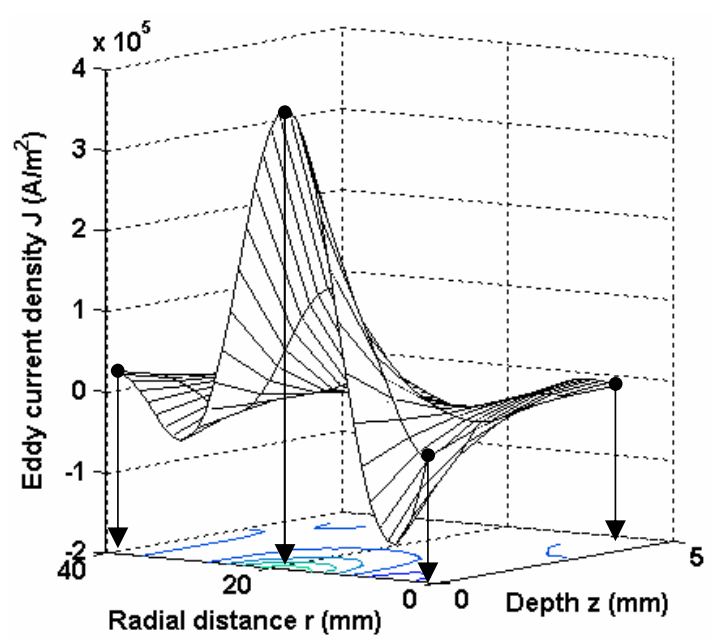

(b) Imaginary component

Fig. 4 Eddy-current distribution for a circular exciting coil parallel to a plane.

component of magnetic flux produced by line $L_{3}$ and $L_{4}$ is counteracted to zero. Along the scanning direction, eddy-current path is distorted by scratch so as to produces the $\mathrm{y}$-component of magnetic flux density $\mathrm{B}$. In the zone without defects the $\mathrm{y}$-component of $\mathrm{B}$ will not appear until defect occurs in this zone. Thus the y-component only represents the scratch information. To utilize the $y$ component of $\mathrm{B}$ as detected signal is prone to cancel the lift-off noise produced by $\mathrm{x}$ - and $\mathrm{z}$-component.

Since SV-GMR sensor exhibits a larger change in resistance for one direction only as shown in Fig. $5^{4)}$, in the principle, the changes of output voltage of the sensor is only derived from scratch. The resistance value of SV-GMR sensor is $400 \Omega$ when there is no any external magnetic field. The sensitivity of resistance is $6.1 \% / \mathrm{mT}$. The DC constant current of $5 \mathrm{~mA}$ is fed to SV-GMR sensor and the lock-in amplifier is used to measure the voltage changes. Besides, since SV-GMR sensor has excellent wide temperature dynamic range $\left(-40-150^{\circ} \mathrm{C}\right)$, 


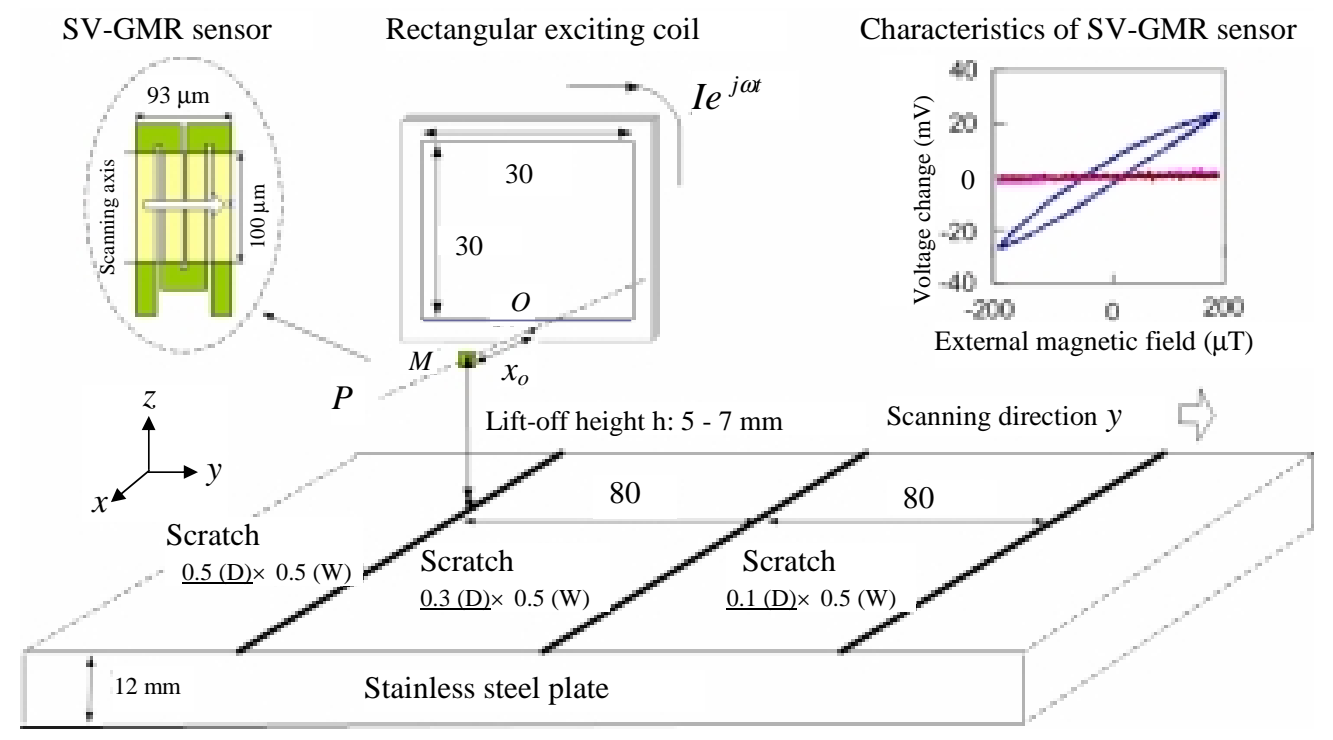

Fig. 5 Fabrication of an ECT probe and tested specimen.

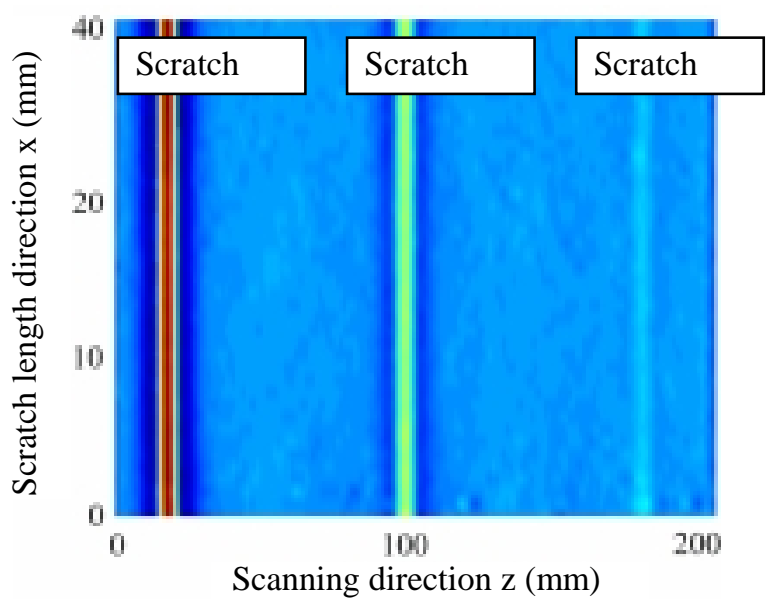

(a) $\mathrm{h}=5 \mathrm{~mm}$

Fig. 6 2-D image results at two lift-off heights.

it is capable to work in the high temperature atmosphere and high frequency detection

\section{Experiment results}

We fabricated the scratch sample of $0.5 \mathrm{~mm}$ width and $0.5,0.3$ and $0.1 \mathrm{~mm}$ depths on the stainless steel plate. Fig. 6 shows the inspected signals of the three scratches with lift-off heights of 5 and $7 \mathrm{~mm}$. We can fabricate a heat insulator in the space to prevent a probe from high temperature stress. The information with respect to the relative depth of three scratches can be clearly acquired at the lift-off height of $5 \mathrm{~mm}$ in Fig. 6 (a). However, it is difficult to obtain scratch information of $0.1 \mathrm{~mm}$ depth at a lift-off height of $7 \mathrm{~mm}$ though scratches with depths of 0.3 - $0.5 \mathrm{~mm}$ are clearly recognized in Fig. 6 (b).

\section{Conclusions}

An analytical solution of a rectangular exciting coil perpendicular to a plane is derived to compare with the

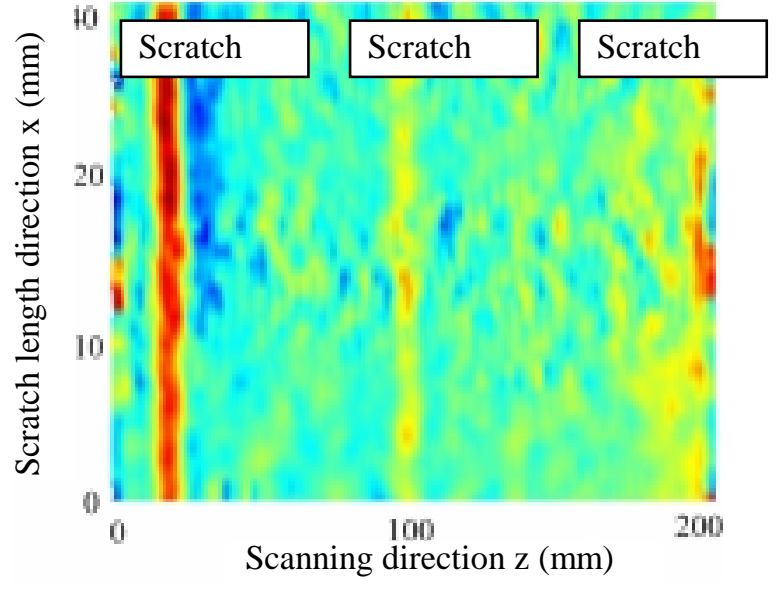

(b) $\mathrm{h}=7 \mathrm{~mm}$

traditional circular coil parallel to a plane. Depending on these solutions and SV-GMR sensor's characteristics, we conclude that the perpendicular style of exciting coil and SV-GMR sensor offers advantages in scratch detection with a high lift-off height. The presented fabrication of ECT probe consisted of a perpendicular exciting coil and SV-GMR sensor is effective in experiments to detect the scratches of $0.1-0.5 \mathrm{~mm}$ depth in a stainless steel plate with lift-off heights of 5 and $7 \mathrm{~mm}$.

\section{References}

1) H.Toyama, S.Yamada, M.Iwahara, D.Roy, K.Miya : JSAEM studies in AEM, 15, 223(2005).

2) J. Juillard, B. de Barmon, and G. Berthiau: IEEE Trans. Magn., 36, 258(2000).

3) C. V. Dodd, and W. E. Deeds: J. Appl. Phys., 39, 2829, (1968).

4) S. Yamada, K. Chomsuwan, Y. Fukuda, M. Iwahara, H. Wakiwaka, and S.Shoji: IEEE Trans. Magn., 40, 2676 (2004).

Received Oct. 19, 2005; Accepted May 19, 2006 